\title{
Influence of gas stunning and halal slaughter (no stunning) on rabbits welfare indicators and meat quality
}

\begin{abstract}
This study assessed the effect of gas stunning which has not been conducted until now in comparison with slaughter without stunning on the welfare and meat quality of rabbits. Eighty male New Zealand White rabbits were divided into two groups of 40 animals and subjected to either halal slaughter without stunning (HS) or gas stunning using $61.4 \% \mathrm{CO}$, $20.3 \%$ oxygen and $18.3 \%$ nitrogen (GS). Analysis of the sticking blood revealed that both slaughter procedures caused a substantial increase in the levels of catecholamines, hypercalcemia, hyperglycemia, lactic acidemia and an increase in enzyme activities. The ultimate $\mathrm{pH}$ of the Longissimus lumborum muscle did not differ between treatments. GS exhibited higher lightness and cooking loss, and lower glycogen and MFI than HS. This indicates that both GS and HS can be significant stressors although the amount of stress may be below the threshold to negatively affect rabbit meat quality.
\end{abstract}

Keyword: Rabbit; Gas stunning; Halal slaughter; Animal welfare; Physiological stress responses; Meat quality 